\title{
A Comparative Study of Image Retrieval Algorithms for Enhancing a Content-based Image Retrieval System
}

\author{
Elsaeed E. AbdElrazek \\ Department of Computer, Preparing Teacher \\ Dumyat University-Faculty of Specific Education \\ Dumyat, Egypt
}

\begin{abstract}
Content Based image retrieval (CBIR) is in retrieve digital images by the actual content in the image. The content are the features of the image such as color, shape, texture and other information about the image including some statistic measures of the image. In this paper Content Based Image Retrieval algorithms are discussed. The comparative study of these algorithms is done. This article covers various techniques for implementing Content Based Image Retrieval algorithms and Some Open Source examples of Content-based Image Retrieval Search Engines
\end{abstract}

\section{General Terms}

Artificial Intelligent-Image processing

\section{Keywords}

Image Retrieval Algorithms, content-based image retrieval system, Feature Detection Algorithms

\section{INTRODUCTION}

With the explosive growth of the Internet, Web Search technology marked by keywords has acquired a great success in the tremendous information retrieval. As the network develops into the Web2.0 era, people no longer satisfy with merely the text-search, also want to be able to find more images from the sample image. In the future, image search engine will become the main tool of the user to retrieve images in the network [1].

The image content is more complexity than the text content to search kinds of information; images can only be expressed through their own content features. Therefore, image retrieval to be implemented is much more difficult than text retrieval.

On the other hand, people have developed many convenient development toolkits, which are capable of establishing image feature database. That makes it possible that the image search technology becomes more and more mature. As the same time, the efficiency of retrieving image becomes better than that of the past [2].

With the growth of the Internet, and the availability of image capturing devices such as digital cameras and image scanners, image databases are becoming larger and more widespread, and there is a growing need for effective and efficient image retrieval systems. There are two approaches for image retrieval: text-based and content-based. The text-based approach can be tracked back to 1970s [3], in such systems, the images are manually annotated by text descriptors, which are then used by a database management system to perform image retrieval. There are two disadvantages with this approach, the first is that a considerable level of human effort is required for manual annotation. The second is the annotation inaccuracy due to the subjectivity of human perception. To overcome the above disadvantages in textbased retrieval system, content-based image retrieval (CBIR) was introduced in the early 1980s.
In CBIR, the image visual content is a matrix of pixel values which are summarized by low-level features such as color, texture, shapes. We describe a CBIR methodology for the retrieval of images, whereas for humans the content of an image refers to what is seen on the image, e.g." a forest, a house, a lake ". One of the research issues in content-based image retrieval is to reduce this semantic gap between the image understanding of humans and the image understanding of the computer, Humans tend to use high-level features (concepts), such as keywords, text descriptors, to interpret images and measure their similarity. While the features are automatically extracted using computer vision techniques are mostly low-level features (color, texture, shape, spatial layout, etc.). In general, there is no direct link between the high-level concepts and the low-level features.

Digital image databases and image processing techniques have developed significantly over the last few years. Today, a growing number of digital image databases are available, and are providing usable and effective access to image collections. In order to access these resources, users need reliable tools to access images. The tool that enables users to find and locate images is an image search engine

Search engines that use Text-Based Image Retrieval (TBIR) are Google, Yahoo. TBIR is based on the assumption that the surrounding text describes the image. The technique relies on text surrounding the image such as filenames, captions and the "alt"-tag in HTML and paragraphs close to the image with possible relevant text. The other approach uses image annotation of the images and is often a manual task. Annotation of images lets the provider annotate the image with the text (metadata) that is considered relevant. Most text based image retrieval systems provide a text input interface that users can type keywords as a query. The query is then processed and matched against the image annotation, and a list of candidate images are returned to the users. The Drawbacks of TBIR as follows [4, 5]:

1) In TBIR, humans are required to personally describe every image in the database, so for a large image database the technique require too much effort and time for manual image annotation.

2) TBIR techniques based on the comparison of the exact string matching. If the query string is misspelled there are no results returned.

3) The manual text annotation is valid only for the language used for the Purpose of annotation. Other people that do not have a background in the used language(s) are not able to use the text-based retrieval systems

4) Description of the image content is subjective to human perception; different people may end up with different descriptions for the content of the image in hand. TBIR is non-standardized because different users use different keywords for annotation.

5) The queries are mainly conducted on the text information and consequently the performance heavily 
depends on the degree of matching between the images and their text description.

6) The use of synonyms would result in missed results that would otherwise be returned.

In order to overcome the drawbacks of text based image retrieval system outlined above, and to assist users in finding desired images from the expected tens of millions of images, the Content-based image retrieval (CBIR) techniques can be designed to meet this aim.

The current research will focus on Comparison of Image Retrieval Algorithms within Image search engines, to identify searchable image features, to compare them based on their features, and to analyze the possible impact of these features on retrieval for enhancing a content-based image retrieval system

Most search engines rely on weak algorithms such as Color Histogram and Texture, which affects search results and images that do not match the query image. So the current research is trying to review these algorithms as an attempt to integrate them to achieve the quality of the search results.

\section{THE RESEARCH PROBLEM CAN BE COUCHED IN THE FOLLOWING QUESTIONS}

1) What Main stages of Web Image Retrieval System?

2) What are the current technologies for image recognition and retrieval on the web?

3) What Image's low level feature algorithms?

4) What are the most important Feature Detection Algorithms?

\section{OBJECTINE}

This search introduces a Comparison of Image Retrieval Algorithms within image search engines on the World Wide Web based on image recognition techniques. The main objectives are summarized in the following aspects:

1) Highlight image retrieval algorithms which collect images from the World Web according to its low level features (color, texture and shape).

2) Forming a scalable and adaptive CBIR framework for World Wide Web (www) users and search engines platforms 2 .

3) Enable the user to search for the images which are similar to his/her query in the contents and returns a set of images that similar to the user's query.

4) Improving the overall performance of feature extracting processing.

5) To acquire reliable and accurate results to validate the approach.

6) Improving the overall timing of user's query.

\section{IMAGE RETRIVAL}

Search for an image from a collection of images was commonly done through the description of the image. As the number of image collections and the size of each collection grow dramatically in recent years, there is also a growing needs for searching for images based on the information that can be extracted from the image themselves rather than their text description. Content Based image retrieval(CBIR) IS an approach for meeting this need .CBIR is in retrieve digital images by the actual content in the image The content are the features of the image such as color, shape, texture and other information about the image including some statistic measures of the image .
Image retrieval techniques integrate both low level Visual features addressing the more detailed perceptual aspects, and high level semantic features underlying the more general conceptual aspects of visual data

Image retrieval can be categorized into the following types [6]:

1) Exact Matching Retrieves: the items that are perfectly matched with the user request which seeks to discover essential commonality of features of two entities being matched

2) Low Level Similarity-Based Searching: Low Level visual features such as color shape. Texture e.g. to represent image content directory

3) Search -by-example is a common practice whereby an image is supplied and the system returns images that have features similar to those of the supplied image .The similarity of images is determined by the values or similarity measures that are specifically defined for each feature according to their physical meaning

4) High Level semantic-Based Searching: the notion of similarity is not based simple feature matching and usually from extended user interaction with the system .at a higher semantic level that is better attuned to matching information needs. Such indexing techniques produce descriptions using a fixed vocabulary or so-called high-level features also referred to as semantic concepts

The image retrieval systems based on the most commonly used image features following [7]:

1) The color: it does not find the images whose colors are exactly matched. But images with similar pixel color information. This approach has been proven to be very successful in retrieving images since concepts of the color-based similarity measure is simple. And the convention algorithms are very easy to implement. Besides, this feature can resist noise and rotation variants in images. However, this feature can only used to take the global characteristics into account rather than the local one in an image. Such as the color difference between neighboring objects in an image.it is often fails to retrieve the images that are taken from the same scene in which the query example is also taken from under different time or conditions

2) The shape: Natural objects are primarily recognized by their shape. A number of features characteristic of object shape are computed for every object identified within each stored image. Generally, Shape representations can be divided into two categories, boundary - based and region-based. The former uses only the outer boundary of the shape while the latter uses the entire shape region .A shape-based image retrieval stein accepts as input an image provided by the user and outputs a set of (possibly ranked) images of the system's database, each of which should contain shapes similar to the query, There are two main types of possible queries: queries by example and quay by sketch. In shape-based retrieval no isolated objects are difficult to deal with because they need to be localized in the image before in order to be compared with the query .shape localization is a non-trivial problem, since it involves high - level scene segmentation capabilities how to separate interesting objects from the background is still an open and difficult research problem in computer vision the second problem is the necessity to deal with inexact matching between a 
stylized sketch and a real . Possibly detailed, shape contained in the image, will be need to take into account possible differences between the two shapes when compared between of them [8].

3) The texture: texture is an important characteristic in many types of images. Despite its importance a formal definition of texture does not exist. When an image has wide variation of tonal primitives, the dominant property of that image is Texture. Texture is the spatial relationship exhibited by grey levels in a digital image. Textural measures are measures capture that spatial relationship among pixels, spatial measures, which refer to measures mostly derived from spatial statistics, have been used largely in geospatial applications for characterizing and quantifying spatial patterns and processes [9].

The method of texture analysis is divided into two approaches: statistical and structural. For biological section images, the statistical approach is appropriate because the image is normally not periodical like a crystal. In the statistical approach, there are various ways to measure the features of the texture. Tested the discriminating power of various tools: spatial gray -level dependence method (SGLDM), gray -level difference method (GLDM),gray-level nun length method(GLNLM), power spectrum method(PSM), Gray level co-occurrence matrix(GLCM),Intensity histogram features and GLCM features are extracted in our proposed method

A useful approach to texture analysis is based on the intensity histogram of all or part of an image. Common histogram features include: moments, entropy dispersion, mean(an estimate of the average intensity level),variance (the second moment is a measure of the dispersion of the region intensity), mean square value or average energy ,skewness (the third moment which gives an indication of the histograms symmetry) and kurtosis (cluster prominence)

One of the simplest ways to extract statistical features in an image is to use the first-order probability distribution of the amplitude of the quantized image may be defined as:

$$
\mathrm{P}(\mathrm{b})=\mathrm{P}_{\mathrm{R}}\left\{\mathrm{F(j,k)=r}{ }_{\mathrm{b}}\right\}
$$

Where rb denotes the quantized amplitude level for $0, \mathrm{~b}, \mathrm{~L}-1$.

The first order histogram estimate of $\mathrm{p}(\mathrm{b})$ is simply

$$
\mathrm{P}(\mathrm{b})=\frac{\mathrm{N}(\mathrm{b})}{\mathrm{M}}
$$

Where $\mathrm{M}$ represents the total number of pixels in a neighborhood window of specified size centered about $(\mathrm{j}, \mathrm{k}), \mathrm{b}$ is a gray level in an image, and $\mathrm{N}(\mathrm{b})$ is the number of pixel of amplitude $r b$ in the same window

\section{CONTENT-BASED IMAGE RETRIVAL (CBIR)}

CBIR is mainly based on the visual content of images such as color, Texture and shape information. Several techniques have been proposed to extract content characteristics from visual data automatically for retrieval proposed. CBIR applications became a part of a practical life and used in several commercial, governmental archives, and academic institutes such as libraries. CBIR is alternative to the text-based image retrieval and becomes the current research area of image retrieval [10, 11]. In CBIR systems, the image content is represented by a vector of image features instead of a set of keyword. The image is retrieved according to the degree of similarity between features of images.

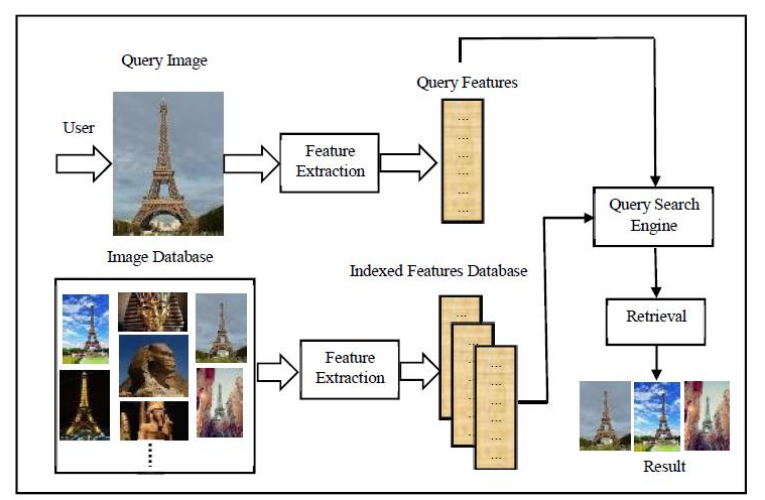

Fig 1: Content-Based Image Retrieval System

The main components of CBIR system are as follows [12]:

1) Graphical User Interface which enable the user to select the query which can an image example: content based image retrieval systems allow the user to specify an image as an example and search for the images that are most similar to it, presented in decreasing order of similarity score.

2) Query / search engine: it is a collection of algorithms responsible for searching the database for images that is similar to the user's query.

3) Image Database: it is repository of images.

4) Feature extraction: it is the process of extracting the visual features (color, shape and texture) from the images.

5) Feature Database: it is repository for image features

\section{FEATURE DETECTION ALGORITHSM}

Feature detection algorithms consist of two basic categories [13]:

1) Feature-based algorithms such as color histogram and shape or edge detector.

2) Texture-based algorithms such as Scale Invariant Feature Transform (SIFT), Speed-Up Robust Feature (SURF) and Principal Component AnalysisSIFT (PCA-SIFT).

\section{COLOR HISTOGRAM AS FEATURE- BASED ALGORITHM}

These algorithms rely on extract a signature for every image based on its pixel values, and to define a rule for comparing images. However, only the color signature is used as a signature to retrieve images. Existing color based generalpurpose image retrieval systems roughly fall into three categories depending on the signature extraction approach used: histogram, color layout, and region-based search. And histogram-based search methods are investigated in two different color spaces. A color space is defined as a model for representing color in terms of intensity values. Typically, a color space defines a one-to four-dimensional space. Threedimensional color spaces such, RGB (Red, Green, and Blue) and HSV (Hue, Saturation and Value), are investigated. 


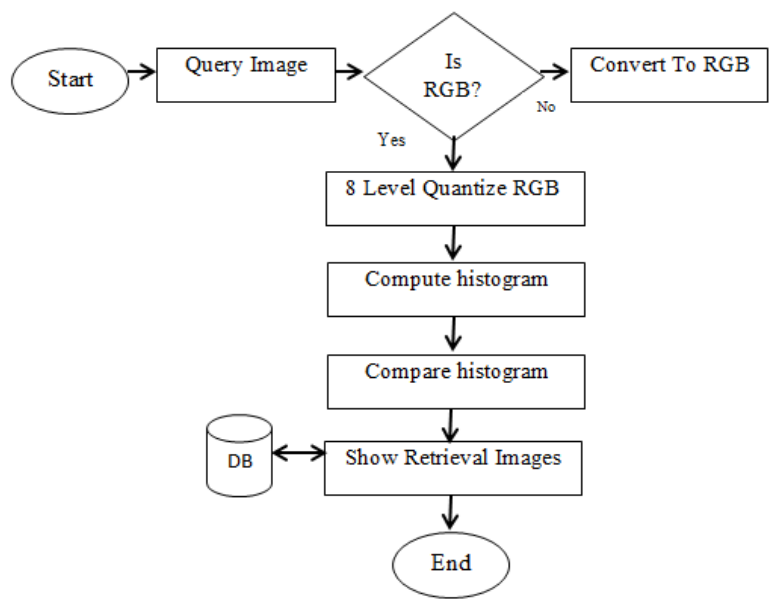

Fig 2: show the flow chart of image retrieval using color histogram [14].

The drawback of a global histogram representation is that information about object location, shape, and texture is discarded. Color Histogram variants with rotation, scale, illumination variation and image noise with no sense of human perception. So, new algorithms are presented to overcome this limitation [3].

\section{FEATURES FROM ACCELERATED SEGMENT TEST (FAST) ALGORITHM}

The beginnings of feature detection can be tracked with the work of Harris and Stephen and the later called Harris Corner Detector which aims to introduce a novel method for the detection and extraction of feature-points or corners.

The Harris corner detector is a popular interest point detector due to its strong invariance to: rotation, scale and image noise by the auto-correlation function. Harris was successful in detecting robust features in any given image meeting basic requirements that satisfied the first two criterions above [15], But since it was only detecting corners, his work suffered from a lack of connectivity of feature-points which represented a major limitation for obtaining major level descriptors (such as surfaces and objects) and limitation in speed .

The main contribution of FAST was summarized as: "A new algorithm which overcame some limitations of currently used corner detectors". With FAST, the detection of corners was prioritized over edges as they claimed that corners are one of the most intuitive types of features that show a strong two dimensional intensity change, and are therefore well distinguished from the neighboring points Also, FAST modified the Harris detector so as to decrease the computational time.[16]

\section{SCALE INVARIANT FEATURE TRANSFORM (SIFT) ALGORITHM}

SIFT was developed by David Lowe in 2004 Aim to presents a method for detecting distinctive invariant features from images that can be later used to perform reliable matching between different views of an object or scene. Two key concepts are used in this definition: distinctive invariant features and reliable matching. SIFT is broken down into four major computational stages $[17,18]$ :

1) Scale-space extreme detection: The first stage of computation searches over all scales and image locations. It is implemented efficiently by using a difference-of-Gaussian function to identify potential interest points that are invariant to scale and orientation

2) Key-point localization: This stage attempts to eliminate more points from the list of key-points by finding those that have low contrast or are poorly localized on an edge.

3) Orientation assignment: One or more orientations are assigned to each key-point location based on local image gradient directions. All future operations are performed on image data that has been transformed relative to the assigned orientation, scale, and location for each feature, thereby providing invariance to these transformations.

4) Key-point descriptor: The local image gradients are measured at the selected scale in the region around each key-point. These are transformed into a representation that allows for significant levels of local shape distortion and change in illumination.

The main contribution of SIFT was summarized as: "A new texture algorithm which invariant feature transforms and overcome some limitations of currently used corner detectors". In SIFT algorithm, "there is no need to analysis the whole image" but you can use only interested key points to describe image. Unfortunately, the drawback of algorithm is that SIFT consider as the slowest texture-based algorithm, complex in computations and consume resources [19].

PCA is a standard technique for dimensionality reduction and has been applied to a broad class of computer vision problems, including feature selection, object recognition. While PCA suffers from a number of shortcomings, such as its implicit assumption of Gaussian distributions and its restriction to orthogonal linear combinations, it remains popular due to its simplicity. The idea of applying PCA to image patches is not novel. Our contribution lies in rigorously demonstrating that PCA is well-suited to representing keypoint patches (once they have been transformed into a canonical scale, position and orientation), and that this representation significantly improves SIFT's matching performance. Research showed that PCA-SIFT was both significantly more accurate and much faster than the standard SIFT local descriptor. However, these results are somewhat surprising since the latter was carefully designed while PCASIFT is a somewhat obvious idea. We now take a closer look at the algorithm [20].

\section{PRINCIPAL COMPONENT ANALYSIS - SCALE INVARIANT FEATURE TRANSFORM (PCA-SIFT ALGORITHM)}

Our algorithm for local descriptors (termed PCA-SIFT) accepts the same input as the standard SIFT descriptor: the sub-pixel location, scale, and dominant orientations of the key-point. We extract a $41 \times 41$ patch at the given scale, centered over the key-point, and rotated to align its dominant orientation. PCA-SIFT can be summarized in the following steps: pre-compute an eigenspace to express the gradient images of local patches; given a patch, compute its local image gradient; project the gradient image vector using the eigenspace to derive a compact feature vector. The feature vector is significantly smaller than the standard SIFT feature vector, and it can be used with the same matching algorithms. 
The Euclidean distance between two feature vectors is used to determine whether the two vectors correspond to the same key-point in different images.

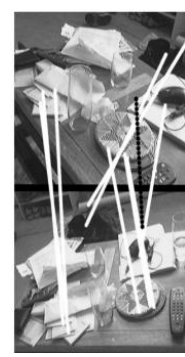

(A1) PCASIFT

$9 / 10$ correct

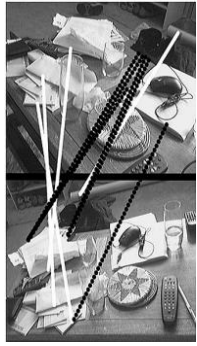

(A2) SIFT

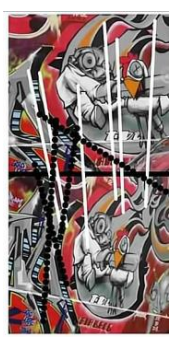

(B1) SIFT

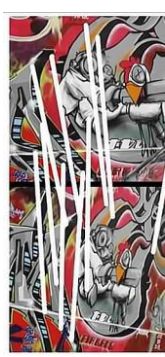

(B2) PCA-

SIFT

$0 / 10$ correct
Fig 3: A comparison between SIFT and PCA-SIFT $(n=20)$ on some challenging real-world images taken from different viewpoints. (A) is a photo of a cluttered coffee table; $(B)$ is a wall covered in Graffiti from the INRIA Graffiti dataset. The top ten matches are shown for each algorithm: solid white lines denote correct matches while dotted black lines show incorrect ones [20].

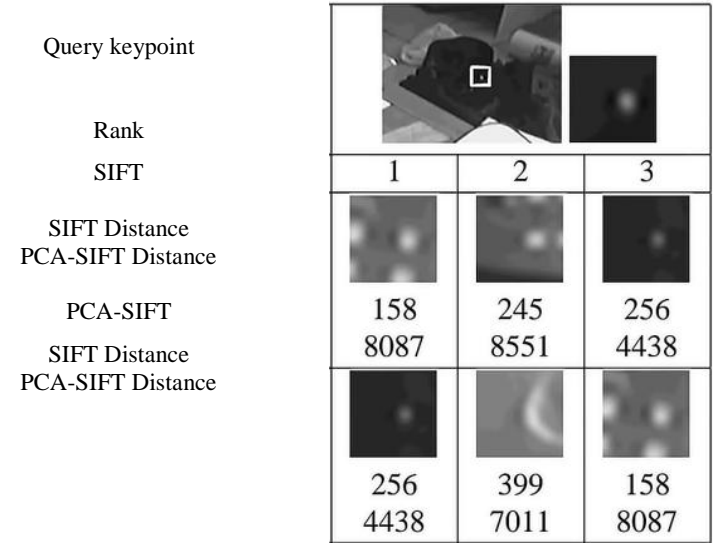

Fig 4: A closer look at matching results for a particular keypoint (zoomed in view of a region from Figure 3). The top three matches for this keypoint for SIFT and PCASIFT $(n=20)$ are shown. The correct match is third on the list for the standard representation, while it is the top match for PCA-SIFT. The two algorithms use different feature spaces so a direct comparison of the distance values is not meaningful [20].

According to PCA-SIFT testing, fewer components requires less storage and will be resulting to a faster matching than SIFT, they choose the dimensionality of the feature space, $\mathrm{n}=20$, which results to significant space benefits. But, PCA suffers from a number of shortcomings, Such as its implicit assumption of Gaussian distributions, less accuracy, less reliable and its restriction to orthogonal linear combinations, it was proved to be less distinctive than SIFT.[19]

The parameters which are used for the experimental evaluation of the results by the above stated algorithms are accuracy, precision and recall [21] where:

Accuracy $=$ Relevant images retrieved in top T returns

Precision $=$ number of retrieved relevant images Total number of retrieved images

Recall $=\quad$ number of retrieved relevant images Total number of relevant images in the database

$\mathrm{T}$ : total number of all relevant images in the database

\section{A COMPARISON OF IMAGE RETRIEVAL ALGORITHMS}

The following table provides the comparison of various Image Retrieval algorithms:

Table 1. The Comparison of Various Image Retrieval Algorithms

\begin{tabular}{|c|c|c|c|c|c|}
\hline No. & Algorithms & Developer & Algorithm Steps & Dataset Used & $\begin{array}{l}\text { Performance } \\
\text { Results }\end{array}$ \\
\hline 1 & $\begin{array}{l}\text { Scale Invariant } \\
\text { Feature } \\
\text { Transform- } \\
\text { (SIFT) }\end{array}$ & $\begin{array}{l}\text { David } \\
\text { Lowe }\end{array}$ & $\begin{array}{l}\text { 1-Constructing a scale space } \\
\text { 2-Laplace of Gaussian approximation 3-Finding } \\
\text { Keypoints } \\
\text { 4-Eliminate edges and low contrast regions5-Assign an } \\
\text { orientation to the keypoints6-keypoint descriptor } \\
\text { extraction } \\
\text { 7-Keypoint Matching }\end{array}$ & $\begin{array}{l}\text { Google Image } \\
\text { API }\end{array}$ & $\begin{array}{l}\text { Precision ranges } \\
\text { from } 80 \% \text { to } \\
85 \%\end{array}$ \\
\hline 2 & $\begin{array}{l}\text { Principle } \\
\text { Component } \\
\text { Analysis- Scale } \\
\text { Invariant } \\
\text { Feature } \\
\text { Transform(PCA- } \\
\text { SIFT }\end{array}$ & $\begin{array}{l}\text { Y. Ke and } \\
\text { R. } \\
\text { Sukthankar }\end{array}$ & $\begin{array}{l}\text { Input: location of keypoint, scale, orientation-Select a } \\
\text { representative set of pictures and detect all keypoints } \\
\text { in these pictures-for each point: Extract an image patch } \\
\text { around it with size } 41 \times 41 \text { pixels, calculate horizontal } \\
\text { and vertical gradients, resulting in a vector of size } \\
93 \times 93 \times 2=3042 \text {-input all these vectors into a } \mathrm{k} \times 3042 \\
\text { matrix A where } \mathrm{k} \text { is the number of keypoints detected - } \\
\text { calculate the covariance matrix of A }\end{array}$ & $\begin{array}{l}\text { Google Image } \\
\text { API }\end{array}$ & $\begin{array}{l}\text { Precision ranges } \\
\text { up to } 90 \%\end{array}$ \\
\hline 3 & $\begin{array}{l}\text { Speeded Up } \\
\text { Robust } \\
\text { Features- } \\
\text { (SURF) }\end{array}$ & $\begin{array}{l}\text { HERBERT } \\
\text { BAY et. all }\end{array}$ & $\begin{array}{l}\text { SURF like SIFT in the major of stages, but different in } \\
\text { two computational stages } \\
\text { a) Orientation Assignment. } \\
\text { b) Descriptor Components }\end{array}$ & $\begin{array}{l}\text { Google Image } \\
\text { API }\end{array}$ & $\begin{array}{l}\text { Precision ranges } \\
\text { up to } 90 \%\end{array}$ \\
\hline
\end{tabular}




\section{SOME OPEN SOURCE OF CONTENT-BASED IMAGE RETRIVAL SEARCH ENGINES 12.1 Alta Vista Photo Finder Search Engine}

Features Similarity is based on visual characteristics such as dominant colors only. No details are given about the exact features. First, the user type keywords to search for images tagged with these words. If a retrieved image is shown with a link "similar", the link gives images that are visually similar to the selected image. Similarity is based on visual characteristics such as dominant colors. The user cannot set the relative weights of these features, but judging from the results, color is the predominant feature

\subsection{Anaktisi Photo Finder Search Engine}

In this website a new set of feature descriptors is presented in a retrieval system. These descriptors have not been designed with particular attention to their size and storage requirements. These descriptors incorporate color information into one histogram while keeping their sizes between 23000 and 740000 bytes per image.

High retrieval scores in content-based image retrieval systems can be attained by adopting relevance feedback mechanisms. These mechanisms require the user to grade the quality of the query results by marking the retrieved images as being either relevant or not. Then, the search engine uses this grading information in subsequent queries to better satisfy users' needs. It is noted that while relevance feedback mechanisms were first introduced in the information retrieval field, they currently receive considerable attention in the CBIR field.

The vast majority of relevance feedback techniques proposed in the literature is based on modifying the values of the search parameters so that they better represent the concept the user has in mind. But, the semantic gap between the user query and the result isn't maintained yet.

There is no ranking algorithm for more usability and reliability Figure 4 shows the result of bus query image of Anaktisi Photo Finder search engine.

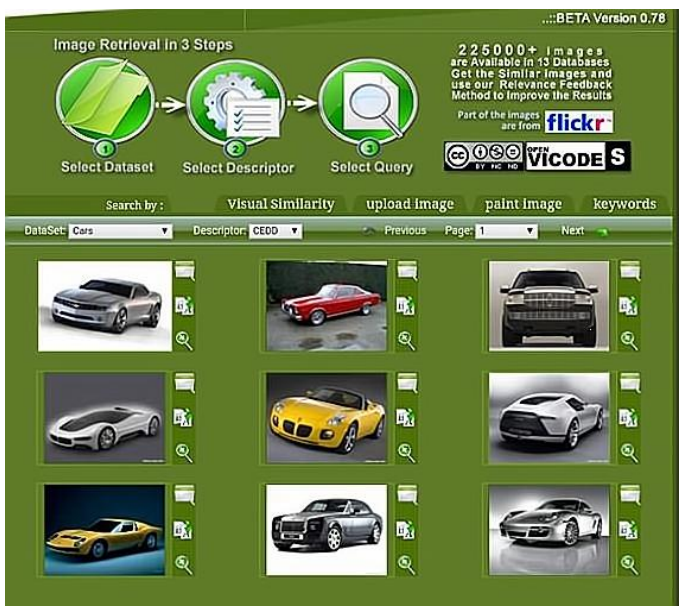

Fig 1: Screenshot about Results of Bus Query Image of Anaktisi Photo Finder Search Engine

\subsection{Akiwi Photo Finder Search Engine}

In this web-site a new set of feature descriptors is presented in a retrieval System. These descriptors have been designed with particular attention to their size and storage requirements, keeping them as small as possible without compromising their discriminating ability. These descriptors incorporate color and texture information into one histogram while keeping their sizes between 22 and 70 kilobytes per image. There are no High retrieval techniques and the semantic gap between human perception and the machine perception is very high. Figure 5 shows the result for logo of University Damietta query image of Akiwi Photo Finder search engine.

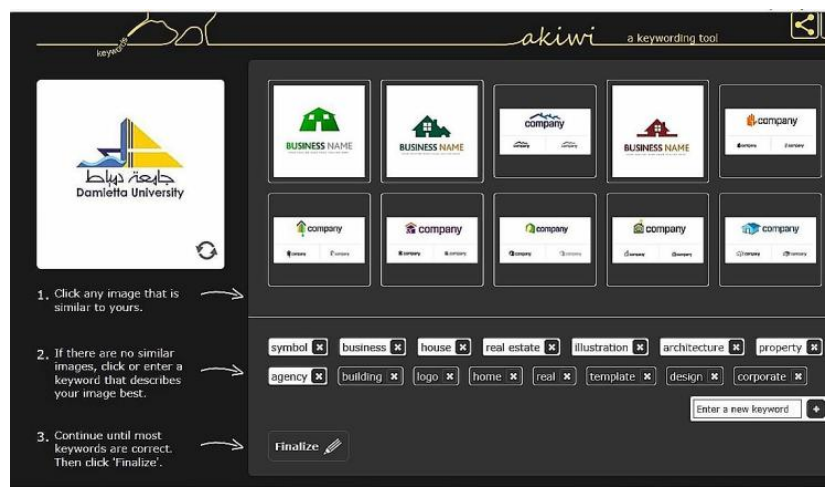

Fig 6: Screenshot about Results for logo of University Damietta Query Image of Akiwi Photo Finder search engine

\subsection{Google Search Engine}

In this web-site, there is no description about what exactly feature extraction algorithm used. But during analysis and testing of Google search, we observe that the result of rose query image returns the exactly image and other images not related to the query.

We considered that returned images by color feature. For semantic technique, Google used ontology tagging for retrieval process. Consequently, ranking method is page rank method as alternative of relevance feedback to optimize usability.

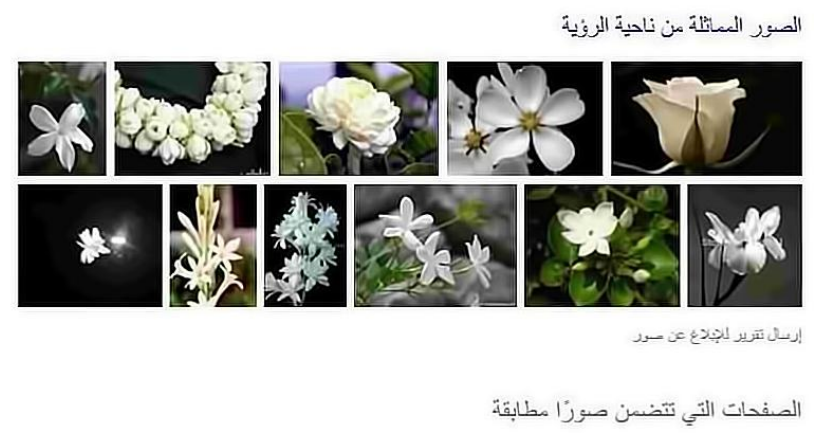

Fig 7: Screenshot about Results of Rose Query Image of Google Search Engine

\section{CONCLUSION AND FUTURE SCOPE}

In this paper, compared to content-based image retrieval algorithms used in the most famous image search engines, the set of algorithms used and their results are discussed in detail. From the results of the different methods discussed, it can be concluded that to improve algorithm retrieval performance must integrate these algorithms to increase the values of standard evaluation criteria such as accuracy, proportion of convergence or accuracy to obtain the higher values of the standard evaluation parameters used to evaluate a large algorithm to demand better results for retrieval performance. 
The horizon is still wide for future studies to work on increasing the accuracy and speed of searching the web. Following points show open issues that need to be addressed:

1) Increase the accuracy of search results by combining of Image Retrieval Algorithms.

2) Increase the accuracy of the search results in the retrieval of images.

3) Increase the speed (Response time) in image retrieval

4) The development of search engines with high accuracy in retrieving information based on the integration of several algorithms of image retrieval.

\section{REFERENCES}

[1] Cao Y,Wang H,Wang C,et al. Mindfinder: interactive sketch-based image search on millions of images $[\mathrm{C}] / /$ Proceedings of the international conference on Multimedia. ACM, PP: 1605-1608, 2010

[2] Wei Z,Zhao P,Zhang Design and implementation of image search algorithm, American Journal of Software Engineering and Applications,3(6):90-94,2014

[3] Y.Liu,D.Zhang and G.Lu"A Survey of Content-Based Image Retrieval with high level Semantics", Pattern Recognition, Vol. 40,PP:262-282,2007

[4] I.King,C.H.Ng,and K.C,Sia,"Distributed Content-Based Visual Information Retrieval System on Peer-to-Peer Networks"ACM TOIS,Vol.22,pp.477-501,2004

[5] H.Q. Nguyen, and Q.T.Ngo:"A Novel Method for Content Based Image Retrieval Using Color Features:"IJCSES International Journal of Computer Sciences and Engineering Systems,Vol.3,no.1,pp.160,2009

[6] Sagarmay Deb,"Multimedia System and Content-based Image Retrieval", Idea Group Inc(IGI),2004

[7] Mehdi Khosrowpour,"Encyclopedia of Information Science and Technology", Idea Group Inc(IGI),2005.

[8] John Eakins,Margaret Graham. "Content-based Image Retrieval ", Jisc Technology,1999

[9] Yu-Jin Zhang, "Semantic -based Visual information Retrieval", Idea Group Inc.(IGI),2007

[10] O.Starostenko,J.Alfredo, and R.Rosas,"Content Based Visual Information Retrieval for Management Information Systems,"Proc
[11] H. L., and G.He,"Shape Feature Extraction of High Resolution Remote Sensing Image Based on SUSA and Moment Invariant",Congress on Image and Signal Processing IEEE,2008

[12] O.Marques, L.M.Mayron,G.BBorba,and H.R.Gamba,"An Attention-Driven Model for Grouping Similar Images with Image Retrieval Applications",EURASIP Journal on Applied Signal Processing,oo.1-16,2007

[13] M.Sahami, V.Mittal,Mayron, S.B.Baluja,S . Rowley "Challenges in Web Information Retrieval", International Conference on Artificial Intelligence, Vol.3157, Berlin, 2004

[14] H.Stokman and T.Gevers."Selection and Fusion of Color Models for Image Feature Detection",IEEE Transactions on Pattern Analysis and Machine Intelligence, Vol.29,PP:371-381,2008

[15] X.Wang."Robust image retrieval based on color histogram of local feature regions", Springer Netherlands, 2009

[16] G. Babbar, Punam Bajaj, Anu Chawla , and Monika Gogna."A Comparative study image matching algorithm", International Journal of Information Technology and Knowledge Management, JulyDecember.pp.337-339, 2010

[17] D. Low. "Distinctive Image Features from ScaleInvariant Key points", University of British Columbia Vancouver, B.C, Canada, International Journal of computer Vision, Vol.60, pp.91-110.2004

[18] R. Hess. "An open source SIFT library ". Proceedings of the International Conference in Multimedia, Italy, pp.1493-1496, 2010

[19] L.Jwun. "A comparison of SIFT, PCA-SIFT, and SURE", International Journal of Image Processing (IJIP), vol. 3, pp: 143-152, 2009

[20] Y. Ke and R.Sukthankar."PCA-SIFT: A More Distinctive Representation for local Image Descriptors", School of Computer Science, Carnegie Mellon University; Intel Research Pittsburgh, Computer Vision and Pattern Recognition, 2004

[21] Wei Bian and Dacheng Tao, "Biased Discriminant Euclidean Embedding for Content-Based Image Retrieval" IEEE Trans.on Image Processing, Vol. 19, No. 2, pp.545-554, Feb 2010 